\title{
Investigating the genetic role of aquaporin 4 gene in migraine
}

\author{
Elisa Rubino $\cdot$ I. Rainero $\cdot$ G. Vaula $\cdot$ \\ F. Crasto $\cdot$ E. Gravante $\cdot$ E. Negro $\cdot$ \\ F. Brega $\cdot$ S. Gallone $\cdot$ L. Pinessi
}

Received: 3 December 2008/ Accepted: 19 January 2009/Published online: 10 February 2009

(C) Springer-Verlag 2009

\begin{abstract}
Aquaporin4 (AQP4) has an important role in water homeostasis of human brain and a dysfunction of AQP4 could induce pathological conditions in neuronal activity. The purpose of our work was to evaluate the association of polymorphisms in the AQP4 gene with the risk and the clinical features of migraine. A total of 293 migraineurs and 249 controls were involved in the study. They were genotyped for four single nucleotide polymorphisms (SNPs) of AQP4 gene. No significant difference in the distribution of AQP4 genotypic and allelic frequencies between cases and controls was found. In addition, haplotype analysis did not show any significant difference. Comparison of the clinical features of the disease according to different AQP4 genotypes showed no significant difference. Our data do not support the hypothesis that the $A Q P 4$ gene could represent a genetic susceptibility factor for migraine.
\end{abstract}

Keywords Aquaporin4 - Migraine without aura . Migraine with aura $\cdot$ Genetic association study

\section{Introduction}

Migraine is a chronic neurovascular disease characterized by recurrent headache attacks, lasting for $4-72 \mathrm{~h}$, associated with nausea and vomiting and accompanied by photophobia and phonophobia [1]. Up to $25 \%$ of migraine sufferers report transient attacks of neurological symptoms,

E. Rubino $(\square) \cdot$ I. Rainero · G. Vaula · F. Crasto · E. Gravante · E. Negro $\cdot$ F. Brega $\cdot$ S. Gallone $\cdot$ L. Pinessi

Neurology II, Department of Neuroscience, University of Turin, Via Cherasco 15, 10126 Turin, Italy

e-mail: elisarubino@inwind.it known as aura, during the headache. Migraine is a public health problem of great impact on both the patient and society. Prevalence studies showed that migraine affects $15-25 \%$ of women and 6-8\% of men [2, 3]. Migraine is rated as one of the most disabling chronic disorders and the annual cost of migraine-related loss in productivity is enormous [4].

Migraine has a strong (up to 50\%) genetic component with a probable multifactorial inheritance [5]. Mutations in three genes encoding neural ion channels have been described in patients with familial hemiplegic migraine (FHM), a rare autosomal dominant form of migraine with aura [6]. However, the success of FHM regarding discovery of genetic defects associated with the disease remains elusive in the common forms of migraine, and causative genes have not been identified yet [7].

Pathophysiological mechanisms underlying migraine are still poorly understood [8]. Recently, a possible alteration of the blood-brain barrier (BBB) in migraine attacks has been suggested [9]. In patients with FHM, a significant BBB opening limited to the cortex and preceding cortical edema was revealed by MRI [10]. In addition, the concentrations of matrix metalloproteinase 9 (MMP-9), a proteolityc zinc-dependent enzyme that disrupts the BBB and promotes brain edema, significantly increased in migraine patients both during the attacks and in the interictal phase [11].

The aquaporins are a family of water-selective membrane channels [12]. Aquaporin 4 (AQP4), the main water channel in the brain, is expressed in pericapillary astrocyte foot processes, glial limiting membranes and ependyma [13, 14].This distribution suggests the involvement of AQP4 in the movement of water between blood and brain and between brain and cerebrospinal fluid, mediating the selective efficient movement of water across the cell 
membrane [15]. AQP4 is involved in BBB development, function and integrity as well [16]. Autoantibodies against AQP4 are detected in the sera of patients affected by neuromyelitis optica, resulting in astrocytes AQP4 loss, and in the typical demyelinating lesions [17]. This protein has also been supposed to be involved in the etiology of cerebral edema and seizures $[18,19]$ and it seems, therefore, a good candidate gene for migraine. Furthermore, the $A Q P 4$ locus is located on chromosome 18q11.2-q12.1 at about $10 \mathrm{cM}$ from the chromosomal regions, suggestive of a linkage to the disease $[20,21]$.

We hypothesized that AQP4 gene polymorphisms would modify the occurrence and the clinical features of migraine. To test this hypothesis, we performed a casecontrol association study in a cohort of Italian migraine patients recruited from a university-based headache clinic and in healthy controls, and we genotyped cases and controls for four different SNPs in the AQP4 gene.

\section{Methods}

\section{Patients and controls}

A total of 293 consecutive migraine patients ( 82 males, 211 females; mean age $\pm \mathrm{SD}=41.2 \pm 13.2$ years, mean age at onset of disease $\pm \mathrm{SD}=18.5 \pm 10.0$ years) attending the Headache Center of the University of Turin (Italy), were involved in the study. The diagnosis of migraine was made according to the International Classification of Headache Disorders (IHCD-II) criteria [22]. For additional statistical analyses, the migraine patients were divided into two groups: migraine without aura (MO; 259 patients) and migraine with aura (MA; 34 patients). A standardized record of all the clinical and psychological characteristics of migraine, suitable for computer analysis, was obtained. Psychological evaluation was performed by a trained psychologist using the Beck Depression Inventory (BDI) and the State and Trait Anxiety Inventory (STAI x-1 and STAI $\mathrm{x}-2)$ tests. A group of 249 sex, age and geographically matched healthy subjects (90 males, 159 females, mean age $\pm \mathrm{SD}=41.9 \pm 12.9$ years) were used as controls. The controls were healthy blood donors and were screened by a neurologist specialized in headaches in order to exclude migraine or other primary headache. Written informed consent was obtained from all participants and the study was approved by the hospital ethics committee.

Genetic analysis

Genomic DNA was extracted from $200 \mu$ l of peripheral blood using the QIAamp DNA mini Kit (QIAGEN). The AQP4 gene consists of five exons spanning a $13.7 \mathrm{~kb}$ region
[23]. Four single nucleotide polymorphisms were genotyped in patients and controls: SNP1 rs3763043 in the $3^{\prime}$ UTR region of the AQP4 gene, SNP2 rs3908956 in exon 5, SNP3 rs35248760 in exon 2, SNP4 rs162008 in the $5^{\prime}$ UTR region. PCR reactions were performed in a final volume of $25 \mu \mathrm{l}$, with $25 \mathrm{ng}$ of genomic DNA, 0.15 unit of Taq Gold DNA polymerase (Applied Biosystems), $125 \mathrm{nM}$ of each primer, $1.5 \mathrm{mM} \mathrm{MgCl} 2$ and $50 \mathrm{mM}$ dNTPs. The following PCR conditions, an initial denaturation at $95^{\circ} \mathrm{C}$ for $5 \mathrm{~min}$ and 35 cycles at $95^{\circ} \mathrm{C}$ for $30 \mathrm{~s}$, specific $T^{\circ}$ annealing for $30 \mathrm{~s}, 72^{\circ} \mathrm{C}$ for $40 \mathrm{~s}$, and a final elongation to $72^{\circ} \mathrm{C} 7 \mathrm{~min}$ were performed. PCR products were electrophoresed on a $1.5 \%$ agarose TBE $1 \mathrm{X}$ gel and stained with ethidium bromide. For all polymorphisms, the amplified fragment was digested with restriction enzymes (MBI Fermentas). The incubation was performed at $37^{\circ} \mathrm{C}$ for $4 \mathrm{~h}$ and the fragments were separated on $2.5 \%$ agarose TBE $1 \mathrm{X}$ gel.

\section{Statistics}

The $\chi^{2}$ test was used to verify the Hardy-Weinberg equilibrium. Statistical analyses were performed using Genepop version 4.0 (http://wbiomed.curtin.edu.au/genepop) and SigmaStat version 3.1 (Jandel Corp., 1994, San Rafael, CA, USA). The distribution of alleles and genotypes was compared using Fisher's exact test and $\chi^{2}$ test. ANOVA followed by Bonferroni correction for multiple comparison was used to compare the clinical characteristics between cases and controls.

Haploview program version 4.1 (www.broad.mit.edu/ $\mathrm{mpg} /$ haploview/) was used for haplotype analysis and for pairwise linkage disequilibrium, $D^{\prime}$ and $r^{2}$. Genetic Power Calculation (http://statgen.iop.kcl.ac.uk/gpc) was used to calculate the power of the association study. According to the recent guidelines for genetic association studies, the level of statistical significance was taken at $P<0.01$ [24].

\section{Results}

All the tested SNPs, but one, were polymorphic. When SNP2 was genotyped, we found only the ancestral allele in both populations. The Hardy-Weinberg equilibrium was verified for all tested populations. The power to detect a significant association between AQP4 and migraine in our study ranged from 0.74 to 0.93 .

The observed genotypic (GF) and allelic frequencies (AF) of the polymorphisms of the AQP4 gene in migraineurs and controls are listed in Table 1. The GF and AF for genotyped SNPs are remarkably similar to that reported in other populations (http://www.ncbi.nlm.nih.gov/SNP). No significant differences were found in the distribution of genotypic and allelic frequencies between cases and 
Table 1 Genotype distribution (GF) and allele frequencies (AF) of the $A Q P 4$ gene in migraine patients and controls

\begin{tabular}{|c|c|c|c|c|c|c|}
\hline & $N$ & GF & & & $\mathrm{AF}$ & \\
\hline AQP4 SNP1 rs376043 & & $A / A(\%)$ & $A / G(\%)$ & $G / G(\%)$ & $A(\%)$ & $G(\%)$ \\
\hline Migraine patients & 293 & $137(46.7)$ & $120(41.0)$ & $36(12.3)$ & $394(67.2)$ & $192(32.8)$ \\
\hline Controls & 249 & $122(49.0)$ & $100(40.2)$ & $27(10.8)$ & $344(69.1)$ & $154(30.9)$ \\
\hline AQP4 SNP3 rs35248760 & & $C / C(\%)$ & $C / A(\%)$ & $A / A(\%)$ & $C$ & $A$ \\
\hline Migraine patients & 293 & $233(79.5)$ & $56(19.1)$ & $4(1.4)$ & $522(89.1)$ & $64(10.9)$ \\
\hline Controls & 249 & $198(79.5)$ & $48(19.3)$ & $3(1.2)$ & $444(89.2)$ & $54(10.8)$ \\
\hline AQP4 SNP4 rs162008 & & $C / C(\%)$ & $C / T(\%)$ & $T / T(\%)$ & $C$ & $T$ \\
\hline Migraine patients & 293 & $193(65.9)$ & $90(30.7)$ & $10(3.4)$ & $476(81.2)$ & $110(18.8)$ \\
\hline Controls & 249 & $153(61.4)$ & $82(32.9)$ & $14(5.6)$ & $388(77.9)$ & $110(22.1)$ \\
\hline
\end{tabular}

$P$ values were calculated by the $\chi^{2}$ test from $3 \times 2$ (genotype) or $2 \times 2$ (allele) contingency tables. No comparison showed significant difference

controls. No gender difference was found in both cases and controls. When patients with migraine were stratified into subgroups (MO and MA), no significant difference was found.

Multilocus haplotypes are usually more informative than any single marker, so we performed a haplotypic analysis with Haploview program version 4.1. So far, the analysis identified a total of seven haplotypes, but we focused the analysis on the five haplotypes with a frequency more than 1\%. A $\chi^{2}$ test of homogeneity turned out not to be significant $(P$ value $>0.05)$, with the highest contribution to the $\chi^{2}$ statistics given by haplotype ACC. The frequency of all haplotypes was similar between the migraineurs and controls. Pairwise analysis showed that the AQP4 SNPs are in LD with each other, in particular for the SNPs rs 35248760 and rs162008 (LOD $=2.94$; Table 2). These results are in accord with other studies concerning LD in AQP4 gene, in particular LOD score more than 2 and $D^{\prime}$ more than 0.7 was found for rs162008 with other two nearby SNPs $[25,26]$.

Finally, we analyzed the following clinical characteristics of the patients: age of onset, duration of the disease, duration of migraine, frequency of migraine, photophobia, phonophobia, nausea and vomiting. The comparison of the clinical characteristics of the disease according to different $A Q P 4$ genotypes showed no significant difference (data not shown).

Table 2 Linkage disequilibrium indices $\left(D^{\prime}\right.$ and $r^{2}$, above and below diagonal, respectively) in the control population

\begin{tabular}{llll}
\hline SNPs & rs376043 & rs35248760 & rs162008 \\
\hline rs376043 & - & 0.617 & 0.389 \\
rs35248760 & 0.021 & - & 1.0 \\
rs162008 & 0.019 & 0.034 & - \\
\hline
\end{tabular}

\section{Discussion}

The present study of an Italian population failed to provide evidence of a genetic association between polymorphisms in the region of the AQP4 gene and migraine. Allelic and genotypic frequencies of the examined polymorphisms were similarly distributed between cases and controls. Furthermore, when the patients were divided into different clinical subgroups (migraine with and without aura), no significant difference in AQP4 gene polymorphism frequencies was found. Haplotype analysis confirmed this lack of association. Finally, different genotypes had no significant effect on the examined clinical characteristics of the disease. So our data do not support the hypothesis that AQP4 is a genetic risk factor for migraine.

The regulation of water balance in the brain is crucial in maintaining neuronal activity. A disruption in this regulation causes an increase in brain water content that significantly contributes to the pathophysiology of a variety of neurological disorders, like brain tumor, abscess, focal ischemia, meningitis and hydrocephalus $[18,19,26-29]$. The discovery of the AQP family of membrane water channels has provided important new insights into the physiology and pathology of brain water homeostasis. At present, 13 mammalian AQP channels (AQP0-AQP12) have been identified. AQP1, AQP4 and AQP8 have an important role in brain water balance and cerebral edema [30, 31]. Several studies in AQP-deficient mice have clarified the role of these membrane water channels in the mechanisms of cytotoxic edema, vasogenic edema and CSF production [28, 32, 33].The ability to modulate water flux through AQP deletion has provided new insights into brain water homeostasis and suggested a number of new research directions. Finally, a potential role for AQP modulators for the therapy of several brain disorders is currently under investigation [34]. So, in consideration of the complex role of these water channels within the central nervous system, 
additional studies are needed to rule out the involvement of aquaporins in migraine pathophysiology.

In conclusion, our data do not support the hypothesis that the $A Q P 4$ gene could represent a genetic susceptibility factor for migraine. Some caution is needed in interpreting the results and additional studies in different population are warranted to confirm our findings.

Acknowledgments The study was supported by a 2006 grant from the "Ministero dell'Università e della Ricerca Scientifica (MURST)" and by a 2007 grant from Regione Piemonte (Italy).

Conflict of interest All authors declared no actual or potential conflict of interest.

\section{References}

1. Ferrari MD (1998) Migraine. Lancet 51:1043-1051

2. Scher AI, Stewart WF, Liberman J et al (1998) Prevalence of frequent headache in a population sample. Headache 38:497-506

3. Stewart WF, Lipton RB, Celentano DD et al (1992) Prevalence of migraine headache in the United States: relation to age, income, race, and other sociodemographic factors. JAMA 267:64-69

4. Burton WN, Conti DJ, Chen CY et al (2002) The economic burden of lost productivity due to migraine headache: a specific worksite analysis. J Occup Environ Med 44:523-529

5. Russell MB, Iselius L, Olesen J (1996) Migraine without aura and migraine with aura are inherited disorders. Cephalalgia 16:305309

6. Estevez M, Gardner KL (2004) Update on the genetics of migraine. Hum Genet 114:225-235

7. Wessman M, Terwindt GM, Kaunisto MA, Palotie A, Ophoff RA (2007) Migraine: a complex genetic disorder. Lancet Neurol 6:521-532

8. Bolay H, Reuter U, Dunn AK et al (2002) Intrinsic brain activity triggers trigeminal meningeal afferents in a migraine model. Nat Med 8:136-142

9. Alvarez-Cermeno J, Gobernado JM, Gimeno A (1986) Transient blood-brain barrier (BBB) damage in migraine. Neurology 26:437

10. Dreier JP, Jurkat-Rott K, Petzold GC et al (2005) Opening of the blood-brain barrier preceding cortical edema in a severe attack of FHM type II. Neurology 64:2145-2147

11. Leira R, Sobrino T, Rodriguez-Yanez M et al (2007) Mmp-9 immunoreactivity in acute migraine. Headache 47:698-702

12. Nielsen S, Nagelhus EA, Amiry-Moghaddam M et al (1997) Specialized membrane domains for water transport in glial cells: high-resolution immunogold cytochemistry of aquaporin-4 in rat brain. J Neurosci 17:171-180

13. Amiry-Moghaddam M, Otsuka T, Hurn PD et al (2003) An alphasyntrophin-dependent pool of AQP4 in astroglial end-feet confers bidirectional water flow between blood and brain. Proc Natl Acad Sci USA 100:2106-2111

14. Abbott NJ, Ronnback L, Hansson E (2006) Astrocyte-endothelial interactions at the blood-brain barrier. Nat Rev Neurosci 7:41-53
15. Nagelhus EA, Horio Y, Inanobe A et al (1999) Immunogold evidence suggests that coupling of $\mathrm{K}^{+}$siphoning and water transport in rat retinal Muller cells is mediated by a coenrichment of Kir4.1 and AQP4 in specific membrane domains. Glia 26:4754

16. Zeng XN, Sun XL, Gao L et al (2006) Aquaporin-4 deficiency down-regulates glutamate uptake and GLT-1 expression in astrocytes. Mol Cell Neurosci 34:34-39

17. Lennon VA, Kryzer TJ, Pittock SJ et al (2005) IgG marker of optic-spinal multiple sclerosis binds to the aquaporin-4 water channel. J Exp Med 202:473-477

18. Binder DK, Yao X, Zador $Z$ et al (2006) Increased seizure duration and slowed potassium kinetics in mice lacking aquaporin-4 water channels. Glia 53:631-636

19. Griesdale DE, Honey CR (2004) Aquaporins and brain edema. Surg Neurol 61:418-421

20. Bjornsson A, Gudmundsson G, Gudfinnsson E et al (2003) Localization of a gene for migraine without aura to chromosome 4q21. Am J Hum Genet 73:986-993

21. Lea RA, Nyholt DR, Curtain RP et al (2005) A genome-wide scan provides evidence for loci influencing a severe heritable form of common migraine. Neurogenetics 6:67-72

22. Headache Classification Subcommittee of the International Headache Society (2004) The international classification of headache disorders, 2nd edn. Cephalalgia 24(Suppl 1):1-151

23. Lu M, Lee MD, Smith BL et al (1996) The human AQP4 gene: definition of the locus encoding two water channel polypeptides in brain. Proc Natl Acad Sci USA 93:10908-10912

24. Bird TD, Jarvik GP, Wood NW (2001) Genetic association studies: genes in search of diseases. Neurology 57:1153-1154

25. Sorani MD, Zador Z, Hurowitz E et al (2008) Novel variants in human aquaporin-4 reduce cellular water permeability. Hum Mol Genet 17:2379-2389

26. Muratake T, Fukui N, Kaneko N et al (2005) Linkage disequilibrium in aquaporin 4 gene and association study with schizophrenia. Psychiatry Clin Neurosci 59:595-598

27. Papadopoulos MC, Verkman AS (2005) Aquaporin-4 gene disruption in mice reduces brain swelling and mortality in pneumococcal meningitis. J Biol Chem 280:13906-13912

28. Bloch O, Papadopoulos MC, Manley GT et al (2005) Aquaporin4 gene deletion in mice increases focal edema associated with staphylococcal brain abscess. J Neurochem 95:254-262

29. Saadoun S, Papadopoulos MC, Davies DC et al (2002) Aquaporin-4 expression is increased in oedematous human brain tumours. J Neurol Neurosurg Psychiatry 72:262-265

30. Benarroch EE (2007) Aquaporin-4, homeostasis, and neurologic disease. Neurology 69:2266-2268

31. Kleffner I, Bungeroth M, Schiffbauer H et al (2008) The role of aquaporin-4 polymorphisms in the development of brain edema after middle cerebral artery occlusion. Stroke 39:1333-1335

32. Manley GT, Fujimura M, Ma T et al (2000) Aquaporin-4 deletion in mice reduces brain edema after acute water intoxication and ischemic stroke. Nat Med 6:159-163

33. Verkman AS, Binder DK, Bloch O et al (2006) Three distinct roles of aquaporin-4 in brain function revealed by knockout mice. Biochim Biophys Acta 1758:1085-1093

34. Papadopoulos MC, Verkman AS (2008) Potential utility of aquaporin modulators for therapy of brain disorders. Prog Brain Res 170:589-601 